\title{
Racial and ethnic variations in caregiving-related physical, emotional, and financial strain during COVID-19 among those caring for adult cancer patients
}

\author{
Margaret L. Longacre ${ }^{1}$ (D) $\cdot$ Melissa F. Miller ${ }^{1} \cdot$ Carolyn Y. Fang ${ }^{2}$ \\ Received: 7 September 2020 / Accepted: 3 December 2020 / Published online: 6 January 2021 \\ (C) The Author(s), under exclusive licence to Springer-Verlag GmbH, DE part of Springer Nature 2021
}

\begin{abstract}
Purpose Cancer caregiving is shown to be a burdensome experience in typical times. The purpose of this study was to describe cancer caregivers' emotional, physical, and financial strain during the COVID-19 pandemic and compared to preCOVID-19, and explore racial and ethnic variations in caregiver strain.

Methods We conducted a cross-sectional online survey using Lucid, LLC, incorporating quotas for race, ethnicity, gender and age. Caregivers had to be adults living in the USA and currently providing unpaid care to an adult cancer patient (i.e., during COVID-19) and prior to the pandemic. We assessed the caregivers' emotional, physical, and financial strain and asked them to compare to preCOVID-19 caregiving. Analyses included descriptive and linear regression adjusting for sociodemographic and caregiving-related variables.

Results A total of 285 caregivers met eligibility, and most were nonHispanic white (72.3\%) and female (59.6\%). Based on a scale of "1: Much lower" to "5: Much higher", the financial, physical and emotional strain/stress experienced by caregivers compared to preCOVID-19 was, on average, 3.52 (SD: 0.82; range: 1-5) for financial strain, 3.61 (SD: 0.86; range: 1-5) for physical strain, and 3.88 (SD: 0.89; range: 1-5) for emotional stress. NonHispanic black caregivers were significantly more likely than nonHispanic white caregivers to indicate that caregiving-related financial strain was higher than preCOVID-19. Moreover, Hispanic caregivers compared to nonHispanic white caregivers reported caregiving-related emotional stress was higher than preCOVID-19.

Conclusion These findings suggest a need to be attentive to racial and ethnic variations in emotional and financial strain and provide targeted support in clinical care and via public policy during a public health crisis.
\end{abstract}

Keywords Caregiving $\cdot$ Cancer $\cdot$ COVID-19 $\cdot$ Emotional and financial strain

\section{Introduction}

Over 1.8 million individuals in the USA are expected to receive a new cancer diagnosis in 2020 and will be in need of acute and ongoing treatment and care [1]. In addition to formal health professionals, relatives, or friends of cancer patients are

Margaret L. Longacre

longacrm@arcadia.edu

1 Department of Public Health, College of Health Sciences, Arcadia University, 214 Brubaker Hall, 450S. Easton Rd., Glenside, PA 19038, USA

2 Fox Chase Cancer Center, Cancer Prevention and Control Program, Philadelphia, PA, USA essential in patient care as they provide many and varied tasks [2]. This cancer caregiving experience is well-known to be demanding, and, as such, most caregivers experience physical and psychosocial health deficits [2-4]. Compared to noncaregivers, caregivers in general are shown to experience poorer physical health $[5,6]$ and psychosocial outcomes, including sleep disruption and heightened emotional stress $[2,5$, 7-9]. Caregivers also report using more emergency health services than noncaregivers and engage in poorer health behaviors (e.g., smoking) [7]. Furthermore, as caregivers provide support to a patient or care recipient, their well-being can have reciprocal implications on the care recipient $[10,11]$.

Due to the impact that COVID-19 has had on many daily activities (e.g., shopping, social interactions), it is likely that COVID-19 is intensifying strain or burden on caregivers. Thus, it is important to assess caregiver well-being during 
crisis, such as the current COVID-19 pandemic, to provide immediate assistance. It is also plausible that caregivers who are members of racial and ethnic minority groups might be more vulnerable to strain given the disproportionate burden of COVID-19 on US racial and ethnic minorities [12, 13]. More specifically, the adverse burden of COVID-19 is especially evident among black and Hispanic individuals $[12,13]$. Such disparities are due in large part to pervasive structural inequalities, including limited access to preventive services and less opportunity and lower ease in complying with physical distancing recommendations because of factors such as higher community density or employment necessity and conditions $[13,14]$. Thus, similar to the trends with COVID-19 in society, it is possible that cancer caregivers' burden during COVID-19 will also vary by race and ethnicity.

The purpose of this study was to characterize caregivingrelated emotional, physical, and financial strain among caregivers for adult cancer patients during COVID-19 and compared to preCOVID-19. A secondary goal was to explore differences in caregiving strain by race and ethnicity. We hypothesize that racial and ethnic minority groups, including black and Hispanic caregivers, will be more likely to express greater caregiving-related physical, financial, and emotional strain/ stress during COVID-19 than nonHispanic white caregivers.

\section{Methods}

\section{Data source}

The primary data source for this analysis was a cross-sectional online survey designed to identify burden and quality of life (QOL) among US adults providing unpaid informal care to an adult relative or friend with cancer. We utilized Lucid, LLC, to implement this survey using Qualtrics and according to specified quota sampling to consider age, gender, race, and ethnicity based on the National Alliance for Caregiving (NAC) and the American Association for Retired Persons (AARP) nationally representative findings of cancer caregivers in the USA [2].

Participants were identified through Lucid, which is a company specializing in identifying samples for academic work. Lucid is a sample "aggregator", meaning they do not own or manage any panel of respondents but rather tap into a variety of respondents across all panels. Lucid's partnering companies find research participants from a diverse array of sources, many of which are double opt-in panels. These companies invite participants to partake in research opportunities through emails, push notifications, in-app pop-ups, or offer walls of engagement opportunities. Their methodology overview and validation of methods is explained previously in the literature [15].

Eligibility was based on the following questions: (1) Which of the following best describes your age? $(<18$ or $\geq 18$ years of age); (2) Which of the following best describes you as a caregiver for someone with cancer? (paid or unpaid); (3) Which of the following best describes the age of your relative or friend with cancer that you are caring for/assisting? $(<18$ or $\geq 18$ years of age); (4) Which of the following best describes you and the care recipient's current US residence? (I live in the USA but the care recipient lives outside of the USA; I live outside of the USA but the care recipient lives in the USA; We both live outside of the USA; We both live in the USA). In light of COVID-19, we added items to the survey on (1) caregiving status prior to COVID-19 and (2) perceptions of their physical, financial, and emotional strain/stress now compared to preCOVID-19. The analytic sample for this primary study was 300 caregivers. The survey was active between May 12 and June 18, 2020. The study was approved by the Arcadia University IRB. All participants provided online informed consent prior to taking the online survey.

\section{Sample}

Eligibility for this analysis included those adults (i.e., $\geq$ 18 years of age) who were providing unpaid care to an adult with cancer during and prior to COVID-19, with both the patient and caregiver residing in the USA. Of the 300 caregivers in the primary sample, 285 met eligibility criteria for this analysis.

\section{Measurement}

\section{Sample characteristics}

Caregivers provided information about gender, age, race and ethnicity, education, employment status, and household income. We also collected information about the caregiving context, including patient's primary cancer site and cancer stage, caregiver's relationship to the care recipient, primary caregiver status, duration of care in years, and hours of care provided per week. See Table 1 for sample characteristics. To better characterize this sample for comparison to existing literature, we included descriptive measures of caregiving burden, distress, and quality of life. These measures included (1) Zarit Burden Interview-12 (ZBI-12), which is shown to be effective in assessing caregiver burden in advanced conditions and oncology [16] with a Cronbach's alpha of 0.88 [17]; (2) National Comprehensive Cancer Network (NCCN) distress thermometer (DT), which has been used to assess distress among caregivers [18-20], and individuals indicate their current level of distress, from 0 (no distress) to 10 (extreme distress); and (3) Caregiver Quality of Life Index (CQLI), which explores emotional, financial, social, and physical quality of life from low (0) to high (100) and has been shown to have high internal reliability (Cronbach alpha of 0.88) [21]. 
Table 1 Sociodemographics and caregiving characteristics among caregivers

\begin{tabular}{|c|c|c|}
\hline Variable & $n$ (or mean) & $\%$ \\
\hline Age, years & $\begin{array}{c}52.7(\mathrm{SD}=14.9) \\
\quad \text { Range: } 20-91\end{array}$ & \\
\hline \multicolumn{3}{|l|}{ Race/ethnicity } \\
\hline NonHispanic White & 206 & 72.3 \\
\hline NonHispanic Black & 27 & 9.5 \\
\hline Hispanic & 39 & 13.7 \\
\hline Other/multiracial & 13 & 4.6 \\
\hline \multicolumn{3}{|l|}{ Gender } \\
\hline Male & 113 & 39.6 \\
\hline Female & 170 & 59.6 \\
\hline Prefer not to answer & 2 & 0.7 \\
\hline \multicolumn{3}{|l|}{ Annual household income } \\
\hline Less than $\$ 20,000$ & 22 & 7.7 \\
\hline$\$ 20,000$ to $\$ 39,999$ & 49 & 17.2 \\
\hline$\$ 40,000$ to $\$ 39,999$ & 44 & 15.4 \\
\hline$\$ 60,000$ to $\$ 39,999$ & 49 & 17.2 \\
\hline$\$ 80,000$ to $\$ 39,999$ & 41 & 14.4 \\
\hline$\$ 100,000$ or above & 78 & 27.4 \\
\hline Prefer not to answer & 2 & 0.7 \\
\hline \multicolumn{3}{|l|}{ Education } \\
\hline Some high school or less & 3 & 1.1 \\
\hline High school graduate or GED & 50 & 17.5 \\
\hline Trade school or associate's degree & 76 & 26.7 \\
\hline College graduate & 92 & 32.3 \\
\hline Postgraduate degree & 64 & 22.5 \\
\hline \multicolumn{3}{|l|}{ Employment status } \\
\hline Full time & 146 & 51.2 \\
\hline Part time & 29 & 10.2 \\
\hline Not employed, retired & 64 & 22.5 \\
\hline Not employed, disability & 21 & 7.4 \\
\hline Not employed, other & 23 & 8.1 \\
\hline Prefer not to answer & 2 & 0.7 \\
\hline \multicolumn{3}{|l|}{ Patient's primary cancer site } \\
\hline Brain & 13 & 4.6 \\
\hline Breast & 61 & 21.4 \\
\hline Bladder & 7 & 2.5 \\
\hline Colon and rectal & 27 & 9.5 \\
\hline Endometrial & 3 & 1.1 \\
\hline Kidney (renal cell) & 5 & 1.8 \\
\hline Leukemia & 15 & 5.3 \\
\hline Lung & 29 & 10.2 \\
\hline Lymphoma & 12 & 4.2 \\
\hline Melanoma & 8 & 2.8 \\
\hline Multiple myeloma & 8 & 2.8 \\
\hline Ovarian & 11 & 3.9 \\
\hline Pancreatic & 10 & 3.5 \\
\hline Prostate & 41 & 14.4 \\
\hline Skin (nonmelanoma) & 4 & 1.4 \\
\hline
\end{tabular}

Table 1 (continued)

\begin{tabular}{|c|c|c|}
\hline Variable & $n$ (or mean) & $\%$ \\
\hline Thyroid & 8 & 2.8 \\
\hline Esophageal/throat/oral & 9 & 3.2 \\
\hline Liver & 4 & 1.4 \\
\hline Missing & 10 & 3.5 \\
\hline \multicolumn{3}{|l|}{ Patient's cancer stage } \\
\hline Stage 0 & 7 & 2.5 \\
\hline Stage I & 32 & 11.2 \\
\hline Stage II & 72 & 25.3 \\
\hline Stage III & 66 & 23.2 \\
\hline Stage IV & 56 & 19.6 \\
\hline $\begin{array}{l}\text { Other or patient's cancer does not have a } \\
\text { stage }\end{array}$ & 29 & 10.2 \\
\hline Do not know & 23 & 8.1 \\
\hline \multicolumn{3}{|l|}{ Caregiver's relationship to the care recipient } \\
\hline Spouse or partner & 93 & 32.6 \\
\hline Parent or parent in-law & 97 & 34.0 \\
\hline Adult child & 6 & 2.1 \\
\hline Friend/neighbor/other relative & 86 & 30.2 \\
\hline Not valid response & 3 & 1.1 \\
\hline \multicolumn{3}{|l|}{ Primary caregiver status } \\
\hline I am the person who provides the most care & 197 & 69.1 \\
\hline I split providing care evenly with one... & 69 & 24.2 \\
\hline Someone else is providing the most care & 19 & 6.7 \\
\hline \multicolumn{3}{|l|}{ Duration of care in years } \\
\hline Less than a year & 52 & 18.2 \\
\hline 1 year to less than 2 years & 81 & 28.4 \\
\hline 2 years to less than 5 years & 111 & 39.0 \\
\hline 5 years to less than 10 years & 26 & 9.1 \\
\hline 10 years or more & 15 & 5.3 \\
\hline \multicolumn{3}{|l|}{ Hours of care provided per week } \\
\hline $0-10 \mathrm{~h}$ & 41 & 14.4 \\
\hline $11-20 \mathrm{~h}$ & 98 & 34.4 \\
\hline $21-40 \mathrm{~h}$ & 70 & 24.6 \\
\hline $41-60 \mathrm{~h}$ & 36 & 12.6 \\
\hline $61-80 \mathrm{~h}$ & 19 & 6.7 \\
\hline $81-100 \mathrm{~h}$ & 12 & 4.2 \\
\hline More than $100 \mathrm{~h}$ & 9 & 3.2 \\
\hline
\end{tabular}

\section{Outcome variables}

Our primary outcomes of interest for this analysis were the caregivers' report of caregiving-related physical, emotional, and financial strain/stress and their perceptions of comparison to preCOVID-19 caregiving. The caregivers' physical, emotional and financial strain/ stress was assessed using the National Alliance for Caregiving and the AARP questions included in their Caregiving in the U.S. national surveys and reports 
$[22,23]$. Participants responded to three questions about financial, physical and emotional stress/strain ("How emotionally/physically/financially straining or stressful is caregiving for you?; Likert response of 1: not at all-5: very much). We also asked caregivers how their current emotional, physical, and financial stress/strain levels compared to preCOVID-19 caregiving. They were asked three separate questions for physical/financial/ emotional strain: "Compared to providing care prior to COVID-19, my [emotional stress/physical strain/financial strain] level now is: 1: much lower; 2: somewhat lower; 3: the same; 4: somewhat higher; or 5: much higher. These questions were developed to mirror the NAC/AARP questions about physical, financial, and emotional stress/strain. A higher rating indicates a greater impact of COVID-19 on caregiving strain/stress.

\section{Analysis}

We conducted descriptive analyses to characterize the caregivers, their caregiving-related burden, distress and QOL, and their financial, physical, and emotional strain/ stress and comparison to preCOVID-19 levels. Fisher's exact test was used to determine if there were nonrandom associations between categorical responses for physical, financial, and emotional strain/stress and race/ethnicity. Confounder-adjusted linear regression models were used to assess associations between the continuous COVID-19 caregiving strain/stress rating and sociodemographic and caregiving characteristics. Unstandardized regression coefficients $(\beta)$ and $95 \%$ confidence intervals $(\mathrm{CI})$ were obtained from linear regression models for each of the strain/stress outcomes. Sensitivity analyses by means of ordered logistic regression models with ordinal outcomes were conducted and showed results similar to linear regression analyses, so we reported results from linear regression for easier interpretation.

A priori-defined covariates included in the analyses were race/ethnicity (nonHispanic white, nonHispanic black, Hispanic, other/multiracial), gender (male, female), age (years), education (did not graduate from college, college graduate, earned a graduate degree), income (intervals of $\$ 20 \mathrm{k}$ ), employment (full-time or part-time, not employed), hours of care provided per week (intervals of 10 or $20 \mathrm{~h}$ ), person providing the most care (no, yes), duration of care $(<1$ year, 1 to $<2$ years, 2 to $<5$ years, 5 to $<10$ years, 10 years, or more), relationship to the care recipient (spouse or partner, parent or parent in-law, adult child, friend/neighbor/ other relative), and patient's primary cancer stage (stage I or II, stage III, stage IV, patient's cancer does not have a stage/DK/other).

\section{Results}

\section{Sample characteristics}

Caregivers are 52.7 years of age on average $(\mathrm{SD}=14.9$; range: 20-91) and predominately nonHispanic white $(72.3 \%)$ and female (59.6\%). $54.7 \%$ had a college degree or higher, most were employed full or part time $(61.4 \%)$ and $58.9 \%$ reported a household income of $\$ 60,000$ or higher. Most caregivers were primary caregivers $(69.1 \%)$ and had provided care less than 5 years $(85.6 \%)$. However, a majority was providing $11-40 \mathrm{~h}$ of care per week (11-20 h: $34.4 \% ; 21-40: 24.6 \%)$. Table 1 presents the sample's sociodemographic and caregiving characteristics. The caregivers' average score on the Zarit Burden Interview-12 was 17.9 (SD: 9.4; range: $0-48)$. The average score on the NCCN distress thermometer was 5.1 (SD: 2.7; range: $0-10$ ) indicating elevated distress levels for the sample on average; $29.8 \%$ had no distress (scores 0-3), 20.7\% mild distress (4 or 5), $28.4 \%$ moderate distress (6 or 7 ), and $21.1 \%$ severe distress ( 8 or higher). Finally, for the CQLI, the overall mean score was 62.86 (SD: 21.4; range: 1-100). Among the CQLI subscales, the emotional domain had the highest (positive) mean score of 65.3 (SD: 23.4; range: 1-100), followed by average scores of 63.5 (SD: 24.3; range: 1-100) in the physical domain, 62.1 (SD: 26.6; range: 1-100) in the financial domain, and 60.5 (SD: 26.4; range: $1-100$ ) in the social domain.

\section{Caregiving-related physical, emotional, and financial strain/stress during COVID-19 compared to preCOVID- 19}

According to the NAC/AARP questions, the caregivers were experiencing moderate financial and physical strain with average scores of 2.76 (SD: 1.33; range: 1-5) and 2.76 (SD: 1.19; range: 1-5), respectively. Emotional stress was higher, on average, with a mean of 3.62 (SD: 1.23; range: 1-5). Moreover, caregivers also expressed high (levels 4 or 5 ) of physical strain (26.7\%), financial strain (30.2\%), and emotional stress $(60.7 \%)$.

Based on a measurement of 1: much lower-5: much higher, the financial, physical, and emotional strain/stress of the caregivers compared to preCOVID-19 was, on average, 3.52 (SD: 0.82; range: 1-5) for financial strain, 3.61 (SD: 0.86; range: $1-5$ ) for physical strain, and 3.88 (SD: 0.89; range: 1-5) for emotional stress. As shown in Figs. 1, 2, and 3 , nearly three-fourths $(71.2 \%)$ of all the caregivers indicated that emotional stress of caregiving was "much higher" or "somewhat higher" currently than when providing care preCOVID-19, while $51.6 \%$ indicated similar level responses for physical strain and $42.1 \%$ for financial strain. 


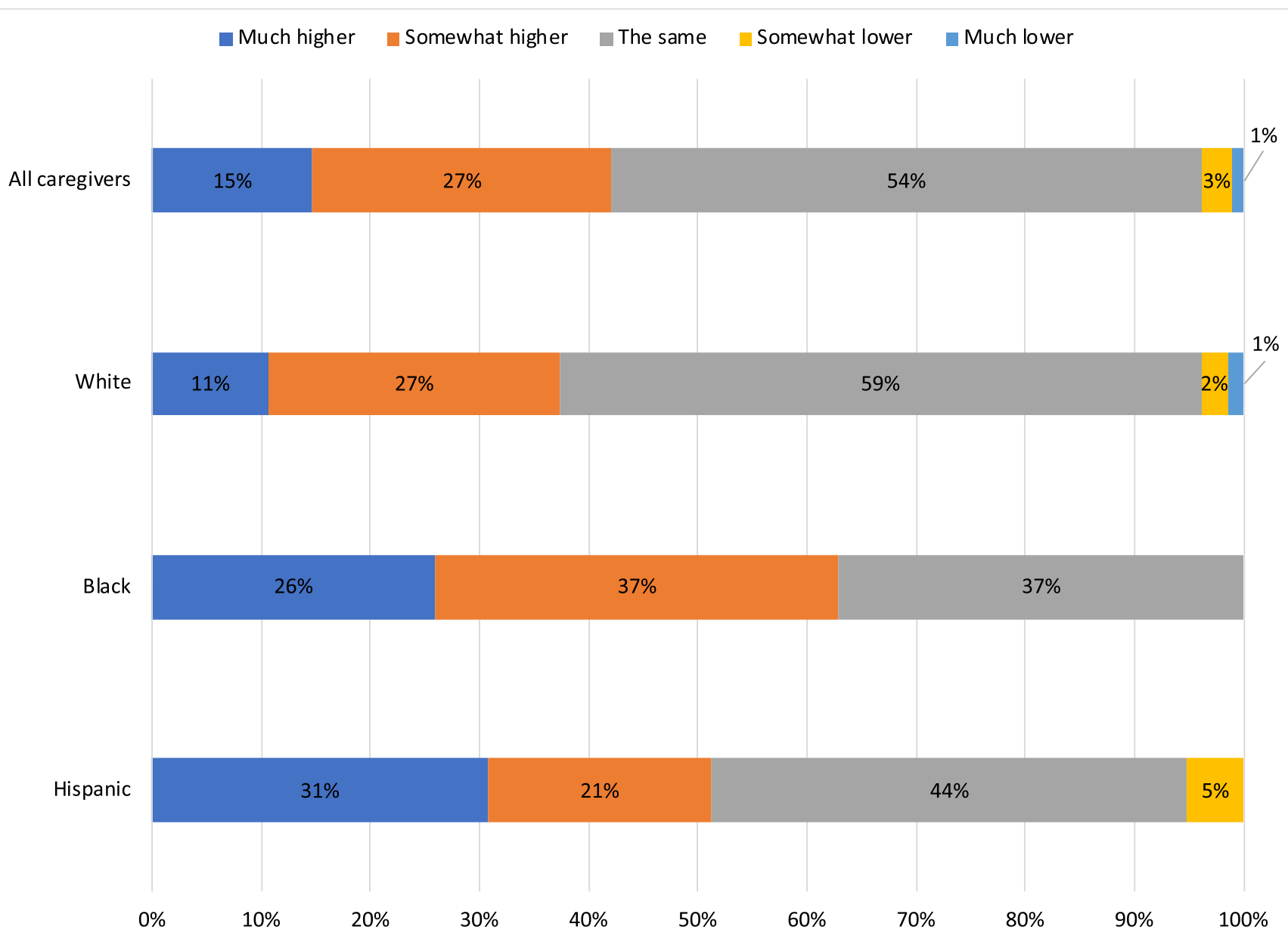

Fig. 1 COVID-19 financial strain compared to preCOVID-19. Fisher's exact test, $p=0.019$

\section{Differences in caregiving-related strain according to race and ethnicity}

A greater proportion of nonHispanic black caregivers reported that caregiving-related financial strain was higher now than prior to COVID-19 (63.0\% total higher; $25.9 \%$ "much higher" and $37.0 \%$ "somewhat higher") compared to other racial/ ethnic groups (Fisher's exact test, $p=0.019$; see Fig. 1). Hispanic caregivers had the highest percentage of caregivers indicating that caregiving-related emotional stress was higher now than prior to COVID-19 (92.3\%; 30.8\% "much higher" and $61.5 \%$ "somewhat higher") compared to other racial/ ethnic group (Fisher's exact test, $p=0.049$, see Fig. 2). A higher percentage of Hispanic caregivers also indicated that caregiving-related physical strain was higher now than preCOVID-19 (66.7\%) compared to other groups, but levels were not significantly different (see Fig. 3).

Multiple linear regression analyses considering all sociodemographic factors and caregiving characteristics indicated statistically significant differences in financial strain and emotional stress according to race/ethnicity. Table 2 model shows that Hispanic caregivers were significantly more likely than non-Hispanic white caregivers to report that the current emotional strain was higher than pre-COVID-19 ( $\beta$ : 0.40; 95\% CI: $0.08,0.72 ; p=0.014)$. Other variables identified as significantly associated with such a perception included female gender $(p=0.028)$, being a college graduate (compared to less education) ( $p=0.021)$, lower income $(p=0.027)$, and more hours of care per week $(p=0.033)$ (see Table 2). As also shown in Table 2, compared to nonHispanic white caregivers, nonHispanic black caregivers reported a significantly greater impact of COVID-19 on caregiving-related financial strain ( $\beta$ : $0.44 ; 95 \%$ CI: $0.11,0.76 ; p=0.009)$. Other variables significantly associated with a perception that caregiving-related financial strain was higher now than preCOVID-19 included lower household income $(p=0.012)$, being unemployed $(p=0.045)$, and higher hours of care provided each week $(p=0.010)$.

\section{Discussion}

Findings from the present study suggest that caregivingrelated strain among cancer caregivers is of concern during 


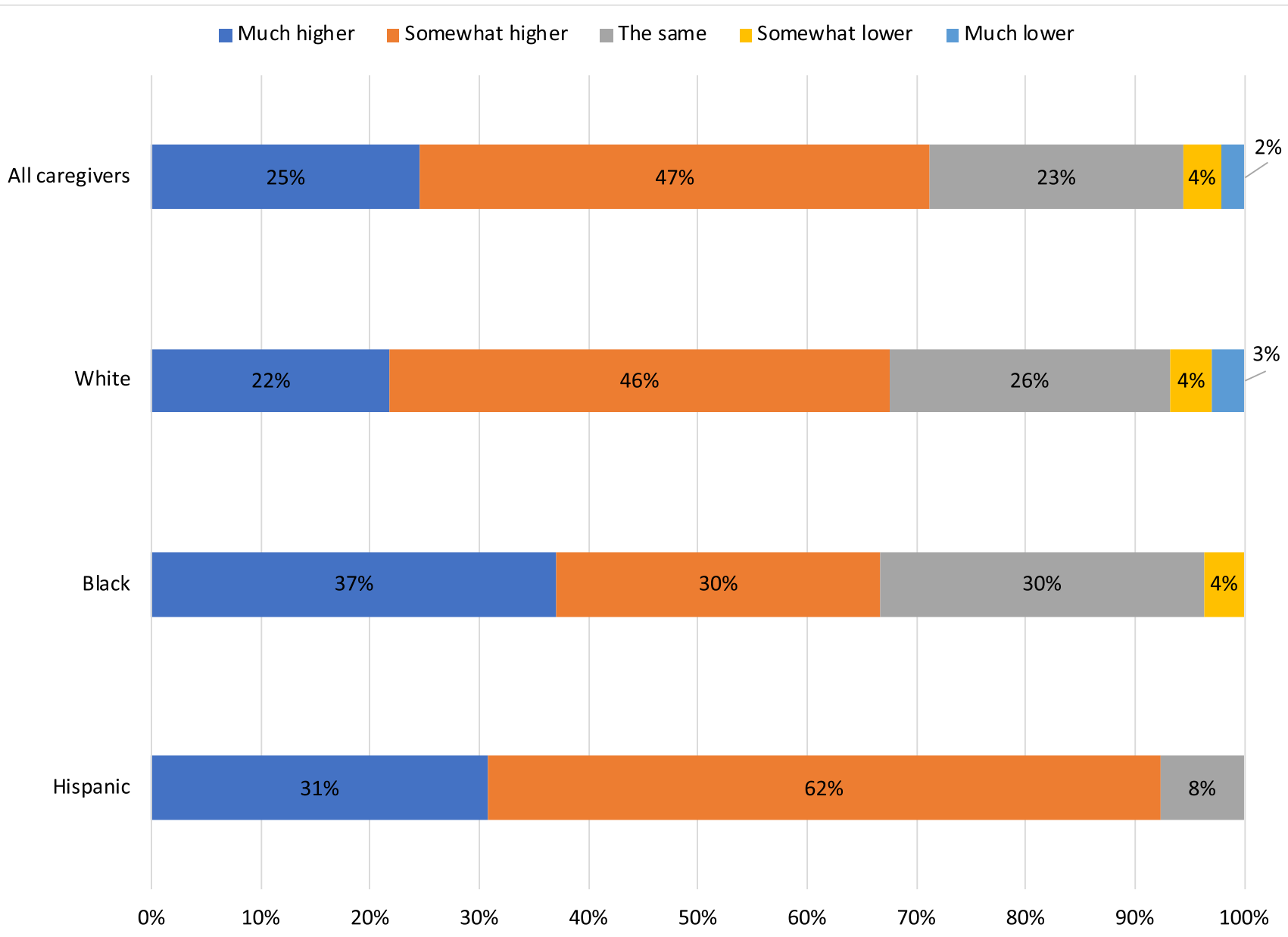

Fig. 2 COVID-19 emotional stress compared to preCOVID-19. Fisher's exact test, $p=0.049$

COVID-19. For example, based on the NAC/AARP questions, financial and physical strain was moderate, while emotional strain was higher. Furthermore, the percentage of cancer caregivers in this sample who expressed high levels (levels 4 and 5) of physical, financial, and emotional strain/stress was higher compared to previous reports of cancer caregivers using the same questions. Specifically, an earlier report by the National Alliance for Caregiving (NAC) on cancer caregivers indicated that $21 \%$ indicated high physical strain, $25 \%$ high financial strain, and 50\% high emotional stress [2]. Our findings (and the NAC findings on cancer caregivers) were also higher in percentage of caregivers indicating high emotional stress and financial strain when compared to noncancer caregivers, which has been previously reported as $17 \%$ for high financial strain and 37\% or high emotional stress (physical strain was not reported) [2].

Similarly, the distress thermometer (DT) findings in this study suggest a high level of distress among the caregivers, with a mean of 5.1 and $49.5 \%$ reporting a distress level of 6 or higher. Of these caregivers expressing scores of 6 or above, $42.6 \%$ were in the range of severe distress ( 8 or above). Caregiving burden was also elevated, as the ZBI-12 mean score in the current sample was higher than that reported for a sample of cancer caregivers providing care for patients with advanced disease [16]. Scoring for quality of life (CQOL) mirrored other findings in terms of suggesting adverse impact of cancer caregiving during COVID-19. The overall CQOL mean was low but slightly higher (better) (62.85) than reported previously among a small sample of cancer caregivers of patients receiving hospice care (61.5) [21]. Furthermore, the CQOL domains for our sample of caregivers ranged from 60.5 to 65.3 , with each being slightly higher (better) than that reported in the literature with exception of the social QOL domain [21]. This finding is particularly relevant given the likelihood of social distancing due to COVID-19, and suggests a need to focus on ways to improve social interactions for caregivers. This might include virtual support groups or encouraging caregivers to connect with others in ways that does not require face-to-face interaction. Though limited, some evidence suggests that online support groups can benefit caregivers in varying contexts (e.g., dementia and oncology) [24, 25]. Finally, the ZBI-12 mean score was higher than that reported for a sample of cancer caregivers providing care for patients with advanced disease [16]. 


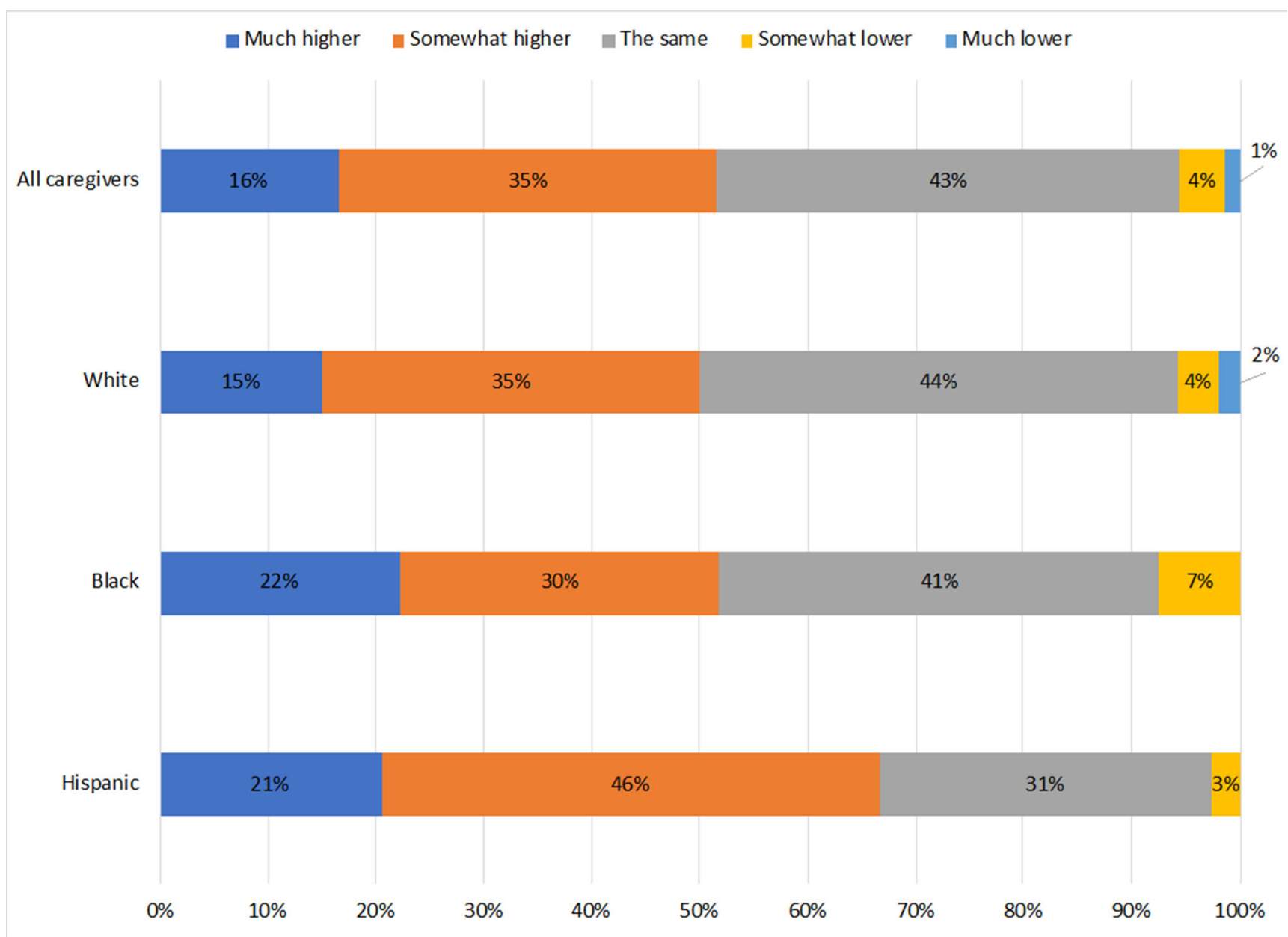

Fig. 3 COVID-19 physical strain compared to preCOVID-19. Fisher's exact test, $p=0.61$

Of even greater concern are our findings that racial and ethnic minority groups are disproportionately experiencing heightened financial strain (nonHispanic black caregivers) and emotional stress (Hispanic caregivers) as cancer caregivers during COVID-19. These findings reflect the broader disparity of COVID-19 within society in that nonHispanic black and Hispanic individuals were more likely to express being adversely impacted $[12,13]$.

Given these high levels of caregiving-related physical, financial, and emotional strain reported during COVID-19 and perception of higher levels compared to preCOVID-19, it is important to consider immediate support. With respect to emotional stress of caregivers, at least one previous report notes that cancer caregivers and noncancer caregivers are rarely asked about their own needs. Specifically, according to findings of a report by the NAC, slightly over half (54\%) of cancer caregivers had been asked by clinicians whether they needed information to care for the patient, and even fewer (29\%) reported being asked if they needed information to care for themselves [2]. Given reduced social interactions due to COVID-19, the clinical context, including virtual, is likely a critical contact point for cancer caregivers to connect with others and receive support. Thus, during this crisis, it seems especially important that the patient's care team consider the well-being of the caregivers by involving the caregiver and assessing his/her needs not only in patient-care but also in selfcare.

Strategies to identify caregivers allow caregivers to convey their needs, and receive support are driven largely by clinical recommendations and state policy. For example, in 2001, the Institutes of Medicine recognized caregivers as an essential part of an older adult's care team [26, 27], and, recently, the National Academy of Medicine (NAM) highlighted a need for person- and family-centered care [28]. These recommendations align with state-level statutes designed to identify and train caregivers at hospital discharge (i.e., the Caregiver Advise, Record, Enable-C.A.R.E-Act) [29]. Furthermore, implementing routine assessment of survivors' needs and caregivers' needs is named as a priority area by Alfano and colleagues [30] in their article on equitably improving outcomes for cancer survivors and supporting caregivers. Though mostly exploratory in terms of acceptability and feasibly assessments, there are increasing examples of clinicbased efforts to assess distress among caregivers in the adult 
Table 2 Results of multivariable linear regression models (unstandardized regression coefficients $(\beta)$ and $95 \%$ confidence intervals)

\begin{tabular}{|c|c|c|c|c|}
\hline \multirow[t]{2}{*}{ Variable } & \multicolumn{2}{|c|}{$\begin{array}{l}\text { Emotional stress }(n=285 ; \\
\left.R^{2}=0.140\right)\end{array}$} & \multicolumn{2}{|c|}{$\begin{array}{l}\text { Financial strain }(n=285 \\
\left.R^{2}=0.141\right)\end{array}$} \\
\hline & $\beta$ & $95 \% \mathrm{CI}$ & $\beta$ & $95 \% \mathrm{CI}$ \\
\hline \multicolumn{5}{|l|}{ Race/ethnicity } \\
\hline NonHispanic White & REF & & REF & \\
\hline NonHispanic Black & 0.24 & $-0.11,0.60$ & $* * 0.44$ & $0.11,0.76$ \\
\hline Hispanic & $* 0.40$ & $0.08,0.72$ & 0.25 & $-0.04,0.54$ \\
\hline Other/multiracial & 0.13 & $-0.36,0.63$ & 0.05 & $-0.41,0.50$ \\
\hline \multicolumn{5}{|l|}{ Gender } \\
\hline Male & REF & & REF & \\
\hline Female & $* 0.25$ & $0.03,0.46$ & 0.18 & $-0.02,0.38$ \\
\hline Age, year & 0.002 & $-0.006,0.01$ & -0.006 & $-0.01,0.002$ \\
\hline \multicolumn{5}{|l|}{ Education } \\
\hline Did not graduate from college & REF & & REF & \\
\hline College graduate & $* 0.31$ & $0.05,0.56$ & 0.17 & $-0.06,0.41$ \\
\hline Earned a graduate degree & 0.06 & $-0.26,0.37$ & 0.09 & $-0.19,0.38$ \\
\hline Income, $\$ 20 \mathrm{k}$ increment categories & $*-0.09$ & $-0.17,-0.01$ & $*-0.09$ & $-0.17,-0.02$ \\
\hline \multicolumn{5}{|l|}{ Employment } \\
\hline Full-time or part-time & REF & & REF & \\
\hline Not employed & -0.09 & $-0.38,0.19$ & $*-0.27$ & $-0.53,-0.006$ \\
\hline Hours of care provided per week, ordinal & $* 0.08$ & $0.01,0.16$ & $* 0.09$ & $0.02,0.16$ \\
\hline \multicolumn{5}{|l|}{ Person providing the most care } \\
\hline No & REF & & REF & \\
\hline Yes & -0.15 & $-0.41,-0.11$ & 0.07 & $-0.17,0.31$ \\
\hline Duration of care in years, ordinal & 0.02 & $-0.09,0.12$ & -0.02 & $-0.11,0.08$ \\
\hline \multicolumn{5}{|l|}{ Relationship to the care recipient } \\
\hline Spouse or partner & REF & & REF & \\
\hline Parent or parent-in-law & 0.06 & $-0.23,0.35$ & -0.10 & $-0.36,0.17$ \\
\hline Adult child & 0.06 & $-0.69,0.80$ & 0.43 & $-0.25,1.11$ \\
\hline Friend, neighbor, or other relative & -0.13 & $-0.44,0.18$ & -0.18 & $-0.46,0.10$ \\
\hline \multicolumn{5}{|l|}{ Patient's primary cancer stage } \\
\hline Stage I or II & REF & & REF & \\
\hline Stage III & -0.06 & $-0.34,0.21$ & -0.09 & $-0.34,0.16$ \\
\hline Stage IV & 0.08 & $-0.21,0.37$ & -0.09 & $-0.36,0.17$ \\
\hline Patient's cancer does not have a stage/DK/Other & -0.19 & $-0.50,0.11$ & -0.21 & $-0.50,0.06$ \\
\hline
\end{tabular}

and pediatric oncology contexts [31-33]. Essential to meeting caregiver needs in clinic-based settings is an organizational shift toward patient and family-centered care [34, 35] and ongoing assessment of caregiver outcomes.

Furthermore, as demonstrated by our findings, engaging caregivers in clinical care might be particularly vital for Hispanic caregivers given their relatively higher levels of emotional stress associated with caregiving during COVID19 than prior. Factors contributing to this finding should be fully explored going forward. For example, in May, 2020, the NAC and AARP provided a snapshot of Hispanic caregivers based on their national data and report on caregivers in the US 2020 Report [22]. This report [36] noted that Hispanic caregivers, compared to nonHispanic whites, tended to be younger and were more often in high intensity care situations. Thus, incorporating communication about caregiving strains and needs within patient clinical interactions might be immediately beneficial for caregivers as a way to uncover factors contributing to elevated stress. Furthermore, this engagement and subsequent response must consider caregivers' cultural beliefs and values. In a recent editorial, Hooper and colleagues [37] noted the importance of recognizing community 
characteristics and providing culturally adapted services. This is an area of ongoing need and response in the clinical and caregiving fields.

Furthermore, we also identified that the financial strain of caregiving during COVID-19 was high for approximately 3 in 10 of these caregivers, and this percentage was higher than previous reports of cancer caregivers and noncancer caregivers [2]. Again, we saw differences by race/ethnicity in that nonHispanic black cancer caregivers were significantly more likely than nonHispanic white caregivers to express that cancer caregiving-related financial strain during COVID-19 (the time of the survey) was perceived to be higher than preCOVID-19. Additional analyses are needed to understand the cause of this financial strain, such as employment strain, lack or loss of health insurance, health care cost even with insurance, or rent or mortgage-related strains. However, given the immediate crisis of COVID-19 and debate on financial support, these findings suggest that certain groups, including caregiving-related groups, might benefit from targeted financial assistance during COVID-19 through public policy efforts.

\section{Limitations}

There are several limitations of this study. First, though online surveying is increasingly being conducted and a recognized strategy to identify nationwide samples quickly and at lower cost, online sampling still presents concerns. One concern with online surveying is an inability to confirm that an individual is indeed a caregiver as indicated. However, we employed several strategies to safeguard against this concern, including, as described in the methods, utilizing several eligibility screening questions that involved several response options as opposed to simply yes or no response with respect to caregiving and study eligibility. We included several eligibility requirements for the study rather than just indicating one was a cancer caregiver. We also assessed duration of time completing the survey and determined that none of the caregivers moved through the survey at what would be considered "speeding," which is measured as completing the survey in a timeframe that is $1 / 3$ of the median time for the sample. The cross-sectional nature of the data is less preferred than a longitudinal design, particularly with respect to comparing their current levels of caregiving-related physical, financial, and emotional stress/strain to preCOVID-19 caregiving. However, as COVID-19 is a crisis event and not something that could have been planned for in terms of study design, such analyses (longitudinal) are not feasible unless a study was already ongoing. Given the ongoing threat of COVID19 , it is suggested that those with access to caregiving data prior to COVID-19 consider conducting follow-up analyses to consider differences, including trend analyses. We also acknowledge that knowing if a patient or caregiver was COVID-19 positive would have provided further context of distress for this analysis. We also acknowledge the relatively small sample size of nonHispanic black and Hispanic respondents. However, these groups represent a good proportion of the sample overall (9.5\% and $13.7 \%$, respectively), and quota sampling was based on national findings of cancer caregivers from a nationally representative sample of caregivers [2].

\section{Conclusion}

Our findings suggest that cancer caregivers are experiencing poorer or heightened strain as a result of COVID-19. Furthermore, this strain/stress is especially evident among non-Hispanic black caregivers (financial strain) and Hispanic caregivers (emotional stress). These findings mirror evidence in society in which racial and ethnic minority groups, particularly black and Hispanic individuals, are more likely to experience adverse outcomes from COVID-19. Given the crisis of this event and lack of time to study longterm, these findings (with aforementioned limitations) should be considered in terms of financial-related public policy to support caregivers as well as organizational (e.g., cancer center) policies to support and further engage caregivers to assess their needs and respond. Our ongoing research will continue to explore how changes in the situation of the caregiver (e.g., loss of employment, difficulties in accessing care) contribute to caregiver outcomes during times of a public health crisis.

Authors' contributions Dr. Longacre contributed to the conceptualization, study design, and facilitation of data collection with Lucid, LLC, data interpretation, and writing of the manuscript. Dr. Miller contributed to the data analysis, interpretation of data, and writing of the manuscript. Dr. Fang contributed to the study design, data interpretation, and writing of the manuscript.

Data availability Data is not publicly available but can be shared as needed with the journal.

\section{Compliance with ethical standards}

All participants provided informed consent, and the study was approved by the Arcadia University IRB.

Conflict of interest The authors declare that they have no conflict of interest.

\section{References}

1. Siegel RL, Miller KD, Jemal A (2020) Cancer statistics, 2020. CA Cancer J Clin 70(1):7-30

2. Hunt GH et al. (2016) Cancer caregiving in the U.S.: an intense, episodic, and challenging care experience. National Alliance for Caregiving 
3. Kent E et al (2016) Caring for caregivers and patients: research and clinical priorities for informal cancer caregiving. Cancer 122(13): 1987-1995

4. Ryn M et al (2011) Objective burden, resources, and other stressors among informal cancer caregivers: a hidden quality issue? Psychooncology 20(1):44-52

5. Bevans M, Sternberg EM (2012) Caregiving burden, stress, and health effects among family caregivers of adult cancer patients. J Am Med Assoc 307(4):398-403

6. Schulz R, Beach SR (1999) Caregiving as a risk factor for mortality: the caregiver health effects study. J Am Med Assoc 282(23): 2215-2219

7. Hopps M et al (2017) The burden of family caregiving in the United States: work productivity, health care resource utilization, and mental health among employed adults. J Multidiscip Healthc 10:437444

8. Kim Y, Schulz R (2008) Family caregivers' strains: comparative analysis of cancer caregiving with dementia, diabetes, and frail elderly caregiving. Journal of Aging and Health 20(5):483-503

9. Stenberg U, Ruland CM, Miaskowski C (2010) Review of the literature on the effects of caring for a patient with cancer. Pyschooncology 19(10):1013-1025

10. Litzelman K, Yabroff KR (2015) How are spousal depressed mood, distress, and quality of life associated with risk of depressed mood in cancer survivors? Longitudinal findings from a national sample. Cancer Epidemiology Biomarkers and Prevention 24(6):969-977

11. Milbury K et al (2013) Longitudinal associations between caregiver burden and patient and spouse distress in couples coping with lung cancer. Support Care Cancer 21(9):2371-2379

12. Webb Hooper M, Nápoles AM, Pérez-Stable EJ (2020) COVID-19 and racial/ethnic disparities. Jama

13. Abedi V et al. (2020) Racial, economic and health inequality and COVID-19 infection in the United States. medRxiv

14. Okonkwo NE et al. (2020) COVID-19 and the US response: accelerating health inequities. BMJ Evid Based Med

15. Coppock A, McClellan OA (2019) Validating the demographic, political, psychological, and experimental results obtained from a new source of online survey respondents. Research and Politics

16. Higginson IJ et al (2010) Short-form Zarit caregiver burden interviews were valid in advanced conditions. J Clin Epidemiol 63(5): 535-542

17. Bedard $\mathrm{M}$ et al (2001) The Zarit burden interview: a new short version and screening version. Gerontologist 41(5):652-657

18. Headley JA, Ownby KK, John LD (2004) The effect of seated exercise on fatigue and quality of life in women with advanced breast cancer. Oncol Nurs Forum 31(5):977-983

19. Fujinami R et al (2015) Family caregivers' distress levels related to quality of life, burden, and preparedness. Psychooncology 24(1): $54-62$
20. Network NCC (2017) NCCN Guidelines for Patients: Distress

21. McMillan SC, Mahon M (1994) The impact of hospice services on the quality of life of primary caregivers. Oncol Nurs Forum 21(7): 1189-1195

22. NAC and AARP (2020) Caregiving in the U.S. 2020 Report. National Alliance for Caregiving AARP

23. NAC and AARP (2015) Caregiving in the U.S. 2015. Washington, D.C.

24. Lee E (2015) Do technology-based support groups reduce care burden among dementia caregivers? A review. J Evid Inf Soc Work 12(5):474-487

25. Benson JJ et al (2020) Online social support groups for informal caregivers of hospice patients with cancer. Eur J Oncol Nurs 44: 101698

26. IOM (2001) Crossing the quality chasm: a new health system for the 21st century. The National Academies Press, Washington DC

27. IOM (2008) Retooling for an aging America: building the health care workforce. The National Academies Press, Washington, DC

28. Wolff JL, Feder J, Schulz R (2016) Supporting family caregivers of older americans. N Engl J Med 375(26):2513-2515

29. Coleman EA (2016) Family caregivers as partners in care transitions: the caregiver advise record and enable act. Journal of Hosptial Medicine 11(12):883-885

30. Alfano CM et al (2019) Equitably improving outcomes for cancer survivors and supporting caregivers: a blueprint for care delivery, research, education, and policy. CA Cancer J Clin 69(1):35-49

31. Longacre ML et al (2018) Reducing informal caregiver burden in cancer: evidence-based programs in practice. Transl Behav Med 8(2):145-155

32. Pierce L et al (2017) Caregiver distress and patient health-related quality of life: psychosocial screening during pediatric cancer treatment. Psychooncology 26(10):1555-1561

33. Shaffer KM et al (2019) Feasibility and acceptability of distress screening for family caregivers at a cancer surgery center. Oncol Nurs Forum 46(2):159-169

34. Frampton SB et al. (2017) Harnessing evidence and experience to change culture: a guiding framework for patient and family engaged care. National Academy of Medicine

35. Carman KL et al. (2014) Roadmap for patient and family engagement in healthcare practice and research. The American Institutes for Research

36. AARP, N. (2020) The "Typical" Hispanic caregiver

37. Webb Hooper M, Nápoles AM, Pérez-Stable EJ (2020) COVID-19 and racial/ethnic disparities. Jama 323(24):2466-2467

Publisher's note Springer Nature remains neutral with regard to jurisdictional claims in published maps and institutional affiliations. 